\title{
Arzthaftung
}

\section{Gutachten sind oft nicht wasserdicht}

\author{
Kommt es zwischen Arzt und Patient zu Streitigkeiten, werden zur \\ Klärung fast immer Gutachter hinzugezogen. Die Qualität der \\ Gutachten ist allerdings umstritten.
}

etabliert. Um zu eruieren, ob tatsächlich ein medizinischer Fehler vorliegt und um ihn als „einfach" oder "grob“ einzustufen, werden in der Regel Gutachten in Auftrag gegeben. „Überwiegend sind das Gutachten des MDK, der Schlichtungsstellen der Ärztekammern oder, sollte der Fall vor Gericht landen, gerichtlich bestellte Sachverständigengutachten“, erklärt Jörg Heynemann, ein auf Arzthaftungsrecht spezialisierter Anwalt aus Berlin.

\section{"Minderwertig und unwissenschaftlich"}

Wie zuverlässig sind Gutachten?

W enn eine medizinische Behandlung nicht erfolgreich war, stellt sich die Frage, ob es ein Behandlungsfehler war. Auseinandersetzungen zwischen Patienten und Ärzten wegen möglicher Behandlungsfehler und damit der Bedarf an medizinischen Gutachten nehmen seit Jahren zu. 2011 haben allein die Gutachter des Medizinischen Dienstes der Krankenversicherung (MDK) 12.686 Behandlungsfehlervorwürfe begutachtet. Landet der Streit vor Gericht, muss der Patient nachweisen, dass der Arzt einen Fehler gemacht hat und dadurch ein Schaden verursacht wurde. Auch mit dem neuen Patientenrechtegesetz, soll sich an der Pflicht des Patienten, den Ärztefehler nachzuweisen, nichts ändern. Nur wenn der Arzt einen groben Behandlungsfehler begangen hat, also wenn er eindeutig und in hohem Maße gegen medizinischen Standard verstoßen hat, kommt es zur Beweislastumkehr (siehe dazu auch URONEWS 11/2012).

Die Vorschriften, die nun in Gesetzesform gegossen werden, sind in der ständigen Rechtsprechung aber schon lange
Da eine Klage vor Gericht mit Kosten verbunden ist, kommt den Schlichtungsstellen der Ärztekammern eine besondere Bedeutung zu. Ihr Schlichtungsverfahren ist für Patienten kostenlos und soll die Streitigkeiten außergerichtlich klären. Sind Patient oder Arzt mit dem Ergebnis nicht einverstanden, könnten sie anschließend immer noch vor Gericht ziehen. Auch Krankenkassen können ihre Versicherten bei der Verfolgung von Schadensersatzansprüchen etwa mit kostenlosen medizinischen Gutachten unterstützten. Nach dem geplanten Patientenrechtegesetz soll aus der Kann- eine Soll-Regelung werden.

Kommt es zu einem Arzthaftungsprozess, haben gerade die medizinischen Gutachter eine sehr hohe Bedeutung: Der Richter ist auf den Sachverstand des Mediziners angewiesen, um in den einzelnen Fällen entscheiden zu können, ob ein Behandlungsfehler tatsächlich vorliegt und welcher Schaden daraus entstanden ist. Das sei angesichts der oft mangelnden Qualität der Gutachten bedenklich, sagt Heynemann. Seinen Worten nach genießen die MDK-Gutachten unter Anwälten und Richtern einen eher schlechten Ruf. „Sie gelten als überwiegend minderwertig und unwissenschaftlich." Abgesehen von den sehr langen Wartezeiten werde oft der vom Patienten dargelegte Sachverhalt mit dem Sachverhalt, der sich aus den Behandlungsunterlagen ergibt, vermengt. Ein solches Gutachten sei wertlos. Auch an den Gutachten der Schlichtungsstellen lässt er kein gutes Haar: „Sie sind qualitativ schlecht und überwiegend ärztefreundlich."

\section{Schwankende Qualität}

Allerdings räumt er ein, dass einige seiner Fachkollegen und Richter den Gutachten der Schlichtungsstellen durchaus gute Qualität mit wenigen negativen Ausreißern attestieren. Iris Harbusch von der Schlichtungsstelle norddeutscher Ärztekammern weist die Vorwürfe des Fachanwalts zurück. „Unsere Gutachter sind unabhängig und an keinerlei Weisungen gebunden", sagt sie. Seit Anfang 2012 vertrete außerdem ein Patientenvertreter die Interessen der Patienten bei der Schlichtungsstelle. Harbusch räumt zwar ein, dass manchmal auch qualitativ schlechte Gutachten vorgelegt werden. Die Schlichtungsstelle, die über eigene Mediziner verfügt, könne aber bei der abschließenden Beurteilung „ein schlechtes Gutachten in eine richtige Entscheidung umwandeln“.

Die gerichtlich bestellten Sachverständigengutachten kommen dabei noch am besten weg, auch wenn Heynemann ihre Qualität als „schwankend“ bezeichnet. In schlechteren Gutachten würden Beweisthemen nicht vollständig abgearbeitet, dafür aber zahlreiche überflüssige Ausführungen angestellt. „Einige Gutachten haben 30 und mehr Seiten und nur ein Bruchteil davon behandelt das eigentliche Problem. Das reicht nicht, um den Sachverhalt ausreichend zu analysieren", erklärt Heynemann. In solchen Fällen müsse das Gericht ein weiteres Gutachten beauftragen. Um die Qualität der Gutachten zu steigern, müssten sich die Sachverständigen streng an Behandlungsunterlagen orientieren, die für die Klärung des Sachverhalts wichtigen Fragen abarbeiten und ihre Einschätzung durch Fachliteratur belegen.

Eugenie Wulfert 